\title{
Graft-versus-host disease management
}

\author{
Mistrik M, Bojtarova E, Sopko L, Masakova L, Roziakova L, Martinka J, Batorova A \\ Department of Hematology and Transfusiology Medical Faculty, Commenius University, Slovak Medical \\ University and University Hospital Bratislava, Slovakia. mistrik@pe.unb.sk
}

\begin{abstract}
Graft-versus-host disease (GVHD) remains a major problem of allogeneic hematopoietic-stem cell transplantation (HSCT) and an obstacle for successful outcome. Clinically significant acute GVHD (grade II or higher) developed in 20 to 65 percent of the patients. Death due to this complication accounts for approximately 50 percent of the deaths that are not due to a relapse of the neoplasm. Up to $70 \%$ of patients who survive beyond day 100 develop chronic GVHD and it is the leading cause of nonrelapse mortality more than 2 years after allogeneic HSCT. In addition, chronic GVHD is associated with decreased quality of life, impaired functional status, and ongoing need for immunosuppressive medications. The incidence of chronic GVHD is increasing because of expansion of the donor population beyond HLA-identical siblings, older recipient age, use of peripheral blood cells as the graft source, and infusion of donor lymphocytes for treatment of recurrent malignancy after HSCT. With the current rush in new findings related to GVHD, we see a significant advancement in its management. Given these various new options and challenges, it is important to identify the minimal requirements for diagnosis and treatment of GVHD, as access to the most sophisticated advances may vary depending on local circumstances (Tab. 4, Fig. 1, Ref. 51). Text in PDF www.elis.sk.

KEY WORDS: allogeneic hematopoietic cell transplantation, acute graft-versus-host disease, chronic graftversus-host disease, graft-versus-host disease, first line graft-versus-host disease treatment, steroid-refractory graft-versus-host disease.
\end{abstract}

Allogeneic hematopoietic stem cell transplantation (HSCT) has evolved into the treatment of choice for a wide variety of hematologic malignancies and non-malignant disorders. Increasing number of HSCT are being performed every year and their indications have expanded in the recent years especially in elderly patients due to the implementation of reduced intensity conditioning (RIC) and in patients without HLA-matched donors owing to the use of cord blood from public banks and haploidentical HSCT. Despite marked improvement in supportive care, immunosuppressive therapy and HLA typing, graft-versus-host disease (GVHD) remains a major cause of peritransplant morbidity and non-relapse mortality, and remains the major obstacle for successful allogeneic stem cell transplantation. Approximately half of the transplanted patients develop clinically significant forms which require therapy, and above $10 \%$ of the patients may die of it. However, GVHD is connected with anti-tumor activity, called graft versus tumor (GVT) or leukemia (GVL) effect, thus suppressing GVHD completely may increase the relapse rate of original disease. GVHD presents with heterogeneous symptoms involving multiple organs including gastrointestinal system, skin, mucosa,

Department of Hematology and Transfusiology Medical Faculty, Commenius University, Slovak Medical University and University Hospital Bratislava, Slovakia

Address for correspondence: M. Mistrik, MD, Department of Hematology and Transfusiology, Medical Faculty, Commenius University, Slovak Medical University and University Hospital Bratislava - Nemocnica sv. CaM Petrzalka, Antolska 11, SK-851 07 Bratislava, Slovakia.

Phone: + 421.2.63532166, Fax: +421.2.63532167 liver and lungs. In the past clinical features developing within 100 days from HSCT were called acute (aGVHD) and those occurring after 100 days were labelled chronic GVHD. This definition has been revised and new categories have been added to the classification such as late-onset aGVHD (acute GVHD occurring after 100 days) and overlap syndrome which includes features of both acute and chronic GVHD (1). And also new definitions of organ system involvement have been introduced (2). Nowadays, the categorization of GVHD is based on combinations of clinical symptoms and the time of onset:

- Classic acute GVHD - cases present within 100 days of HSCT and display features of acute GVHD. Diagnostic and distinctive features of chronic GVHD are absent.

- Persistent, recurrent, late onset acute GVHD - cases present more than 100 days post-HSCT with features of acute GVHD. Diagnostic and distinctive features of chronic GVHD are absent.

- Classic chronic GVHD - cases may present at any time postHSCT. Diagnostic and distinctive features of chronic GVHD are present. There are no features of acute GVHD.

- Overlap syndrome - cases may present at any time post-HSCT with features of both chronic GVHD and acute GVHD. On occasion, this is informally referred to as ,acute on chronic“" GVHD.

The incidence of aGVHD varies with incidence of grade IIIV at $40 \%$ in matched related donor (MRD) transplant to $50 \%$ in matched unrelated donor (MUD) transplant. Risk factors for aGVHD include degree of HLA disparity, donor and recipient 
gender disparity (female donor to male recipient), intensity of the transplant conditioning regimen, patient age, source of graft, previous alloimmunization of the donor and the type of GVHD prophylaxis. However, risk factors differ by underlying disease, requiring distinct risk models for each condition. The incidence and severity of aGVHD also appears to increase with pre-transplant comorbidities. About 30-70 \% of allogeneic HSCT recipients alive after 100 days develop cGVHD, and it is the leading cause of late death. One should expect increase in acute and chronic GVHD incidence in the future due to increasing use of mobilized peripheral blood graft, and unrelated and/ or mismatch HSCT (3). Despite the infusion of HLA class I and II disparate grafts, the incidence and severity of acute and chronic GVHD among unrelated umbilical cord blood recipients has thus far been lower than previously reported in recipients of matched unrelated donor marrow or partially-matched family member marrow allograft.

\section{GVHD pathophysiology}

Host antigen presenting cells (APCs) activation leads to donor $\mathrm{T}$ cells proliferation, differentiation and migration leading to destruction of target tissues (Fig. 1). Initial step in aGVHD is the activation of host APCs, which is mediated by the underlying disease process and the conditioning regimen through tissue damage. The damage to host tissues leads to production of chemokines and cytokines (tumor necrosis factor - TNF $\alpha$, interleukin - IL-1, -2 and -6 ), and increased expression of major histocompatibility complex (MHC) antigens, adhesion molecules and costimulatory molecules in the tissue. The injury to the gastrointestinal tract from the conditioning regimen causes translocation of proinflammatory stimuli (such as bacterial lipopolysaccharide) that activate host APCs. In the second step the donor T cells are activated by
APCs and then differentiate and proliferate. The first interaction between APCs and T cells takes place in the lymphoid tissues associated with gastrointestinal (GI) tract (Peyer's patches). T cell activation is aided by costimulatory molecules on the surface of APCs. In HLA identical HSCT the GVHD is produced by CD4+ and $\mathrm{CD} 8+$ cells in response to minor histocompatibility antigen differences. Activation of the immune cells leads to transcription of genes leading to increased production of cytokines and their receptors. The effector phase in the aGVHD pathogenesis involves cytotoxic $\mathrm{T}$ cells. Chemokines direct $\mathrm{T}$ cell migration to the target organs where they cause damage. Expression of integrins and their respective ligands plays an important role in homing of donor $\mathrm{T}$ cells to Peyer's patches during aGVHD. The GI tract is especially susceptible to damage from TNF $\alpha$ and the GI tract play a major role in generation of the cytokine storm that is the characteristic of aGVHD (4). TNF $\alpha$ can be produced by both donor and host cells and produces myriad of effects including activation of APCs and alloantigen presentation, localization of immune effector cells to the target organs via increased chemokine production and causing direct tissue necrosis.

The pathophysiology of cGVHD is more complex. All the above mentioned mechanisms are relevant as well as other potential pathways. Thymic dysfunction caused by aGVHD has been implicated in the development of cGVHD. A newer role of B cells including immune regulation and immunostimulation via antigen presentation has been recognized in development of cGVHD (5). Antibodies to platelet-derived growth factor (PDGF) receptor, to extracellular matrix protein 1 and to $\mathrm{Y}$ chromosome mHA have been found in GGVHD patients, the levels of which are shown to be reduced with rituximab therapy (6). Multiple other auto and allo antibodies have been identified in patients with cGVHD, but the clear function of these antibodies in pathogenesis of cGVHD as

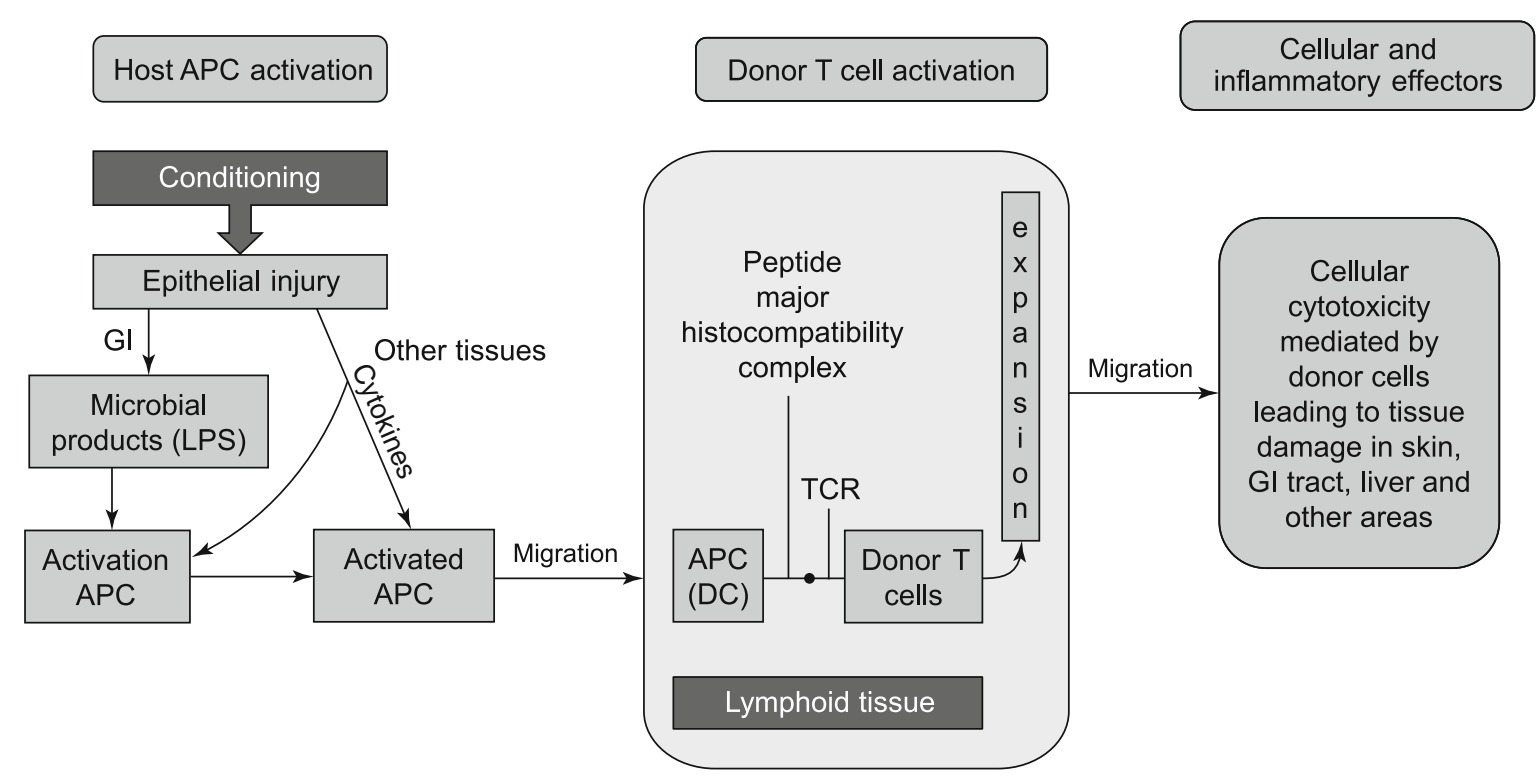

Fig. 1. A three-step GVHD pathogenesis. APC: antigen presenting cell; DC: dendritic cells; LPS: lipopolysaccharides; TCR = T cell receptor. 
they have in other autoimmune diseases is unclear, and they possibly represent immune dysregulation which is a hallmark of GVHD.

Regulatory T Cells (CD4+, CD25+) (Tregs) play important roles in the modulation of GVHD, and Treg deficiency is in cGVHD patients (7). Tregs suppress proliferation and function of TH1 cells which are the main effector of GVHD (8). Donor CD4+ $\mathrm{T}$ and $\mathrm{B}$ cells are essential for development of cGVHD. Early donor dendritic cell reconstitution is associated with decreased incidence of severe GVHD (9). From day 100 onwards after allogeneic HSCT the persistence of host dendritic cells correlates with onset of severe GVHD. Regulatory dendritic cells have a protective effect against cGVHD which is mediated by generation of alloreactive Tregs (10). The upregulation of natural killer cells (NK cells) is associated with reduced incidence of GVHD (11).

\section{Acute GVHD clinical and histological manifestations}

Acute GVHD classically presents in the early post-transplantation period. The initial signs and symptoms of aGVHD most commonly occur around the time of white blood cell engraftment. Although initial definitions of aGVHD required an onset of symptoms before 100 days post transplantation, the current National Institutes of Health (NIH) consensus criteria use clinical findings, rather than a set time period, to differentiate between acute and chronic GVHD. As such, patients presenting with typical findings of aGVHD prior to day 100 are considered to have "classic acute GVHD," whereas patients presenting with the same findings after day 100 , typically upon reduction of immunosuppression, are categorized as having "late onset acute GVHD". Some clinicians also use the terms "early onset acute GVHD" or "hyperacute GVHD" to describe symptoms of aGVHD occurring within 14 days of transplant.

The immune system, skin, gastrointestinal tract, and liver are the principal target organs in patients with acute GVHD. In most patients, the first most common clinical manifestation of acute GVHD is skin involvement, a maculopapular rash, usually occurring at or near the time of the white blood cell engraftment. The rash initially involves the nape of the neck, ears, shoulders, the palms of the hands, and the soles of the feet. It can be described as a sunburn and may be pruritic or painful. From these initial areas of presentation, the rash may spread to involve the whole integument, eventually becoming confluent. In severe GVHD, the maculopapular rash forms bullous lesions with toxic epidermal necrolysis mimicking Stevens-Johnson syndrome. Histologic examination of the skin reveals changes in the dermal and epidermal layers. Characteristic findings include exocytosed lymphocytes, dyskeratotic epidermal keratinocytes, follicular involvement, satellite lymphocytes adjacent to or surrounding dyskeratotic epidermal keratinocytes, and dermal perivascular lymphocytic infiltration. The most consistent histologic feature is individual cell death (apoptosis) at the base of crypts. However, similar changes can result from cytotoxic therapy used in the preparative regimen for $\mathrm{HCT}$, and bacterial or viral infections or reactivations.

Acute GVHD frequently involves both the upper and lower gastrointestinal tract. Gastrointestinal involvement usually pres- ents with diarrhea and abdominal pain, but may also manifest as nausea, vomiting, and anorexia. Confirmation of the diagnosis is provided by pathologic evaluation of tissue obtained by upper endoscopy, colonoscopy. And most cases of acute GVHD of the gastrointestinal tract can be identified by rectal biopsy. However, a negative rectal biopsy does not rule out gastrointestinal GVHD. Further evaluation with upper endoscopy or colonoscopy should be performed for patients with clinical symptoms suggestive of gastrointestinal involvement in the setting of a negative rectal biopsy.

The diagnosis of gastrointestinal involvement requires pathologic evaluation of the tissue.

Involvement of the lower gastrointestinal tract with acute GVHD is often severe, and is characterized by diarrhea, with or without hematochezia, and abdominal cramping. Confirmation of the diagnosis is performed by pathologic evaluation of tissue obtained by rectal biopsy or colonoscopy. Patients with acute GVHD can develop severe diarrhea, occasionally exceeding 10 liters a day. The stool may initially be watery, but frequently becomes bloody. Maintenance of adequate fluid balance may be extremely difficult in such patients. The blood loss can result in significant transfusion requirements. It is not unusual for patients to require frequent transfusions of packed red blood cells per day to maintain a stable hematocrit. The diarrhea is secretory and characteristically continues despite fasting and occurs day and night. It can be accompanied by crampy abdominal pain that can also be difficult to manage. Severe ileus may develop in association with acute GVHD or result from increased opioid use required to control the physical discomfort.

From a diagnostic viewpoint, diarrhea, independent of the presence of acute GVHD, is a common occurrence following HSCT. During the first weeks, diarrhea may be due to the preparatory regimen or to the administration of nonabsorbable or systemic antibiotics. Later, superinfection and Clostridium difficile-associated diarrhea must be taken into consideration as possible causes. Radiologic findings are not diagnostic of GVHD, but if performed for other reasons may show luminal dilation with thickening of the small bowel wall (called a ,ribbon sign“) and air or fluid levels suggestive of ileus. On endoscopy, acute GVHD manifests as spotted erythema, aphthous lesions, and denudation of the mucosa. While acute GVHD of the intestine may be suggested by changes on endoscopy, pathologic evaluation of tissue is required for the diagnosis and visually normal mucosa does not eliminate the possibility of involvement.

A rectal biopsy is usually helpful in making the diagnosis of acute GVHD affecting the gastrointestinal tract. On histologic examination, crypt cell necrosis is observed with accumulation of degenerative material in the dead crypts. With severe disease, whole areas may be denuded with total loss of the epithelium, a finding similar to that observed in the skin. Colonoscopy or upper endoscopy is usually also performed. Infection of the gastrointestinal tract, principally with cytomegalovirus, may mimic the clinical and histologic features of acute GVHD. As a result, selective staining for such pathogens should be performed on the biopsy specimen.

Involvement of the upper gastrointestinal tract with acute GVHD often presents with anorexia, dyspepsia, food intoler- 
ance, nausea, and vomiting. Patients may also display gingivitis and mucositis, although these findings are more common due to the effects of conditioning regimens. The diagnosis is verified by positive upper endoscopic biopsies of the esophagus and stomach. The differential diagnosis includes herpes simplex virus or candida esophagitis, gastritis, peptic ulcers, and gastrointestinal toxicity due to chemotherapy and/or radiation.

Acute GVHD affecting the upper gastrointestinal tract appears to be more responsive to immunosuppressive therapy than involvement of other areas of the gut. Patients with upper gastrointestinal disease who fail treatment progress to symptomatic lower gastrointestinal involvement, which suggests that this syndrome may be an earlier form of intestinal pathology. Initial studies suggest that patients with steroid refractory gastrointestinal tract involvement have expanded $\mathrm{T}$ cell clones within the gastrointestinal tract.

Liver involvement usually presents in patients with signs of cutaneous and/or gastrointestinal acute GVHD. Rarely, patients have moderate to severe hepatic GVHD without evidence of other organ involvement. Although liver involvement may be suggested by abnormalities in liver function tests in the setting of cutaneous or gastrointestinal GVHD, liver biopsy is required to document GVHD of the liver.

Hepatic involvement is manifested by abnormal liver function tests, with the earliest and most common finding being a rise in the serum levels of conjugated bilirubin and alkaline phosphatase. Serum cholesterol is usually elevated, while coagulopathy and hyperammonemia are very rare but may develop in severe cases. Patients may also demonstrate painful hepatomegaly, dark urine, pale stool, fluid retention, and pruritus. Fever, anorexia, and nausea are common nonspecific symptoms.

The abnormalities in liver function tests reflect the pathology associated with liver GVHD: damage to the bile canaliculi, leading to cholestasis. Temporary dilatation of the common bile duct has been described in this setting. However, a rise in the serum concentration of bilirubin or alkaline phosphatase is nonspecific. In this setting, the most common confounding disorders include:

- Hepatic sinusoidal obstructive syndrome (also known as hepatic veno-occlusive disease) is a relatively common toxicity associated with the use of high dose therapy.

- Hepatic infections (primarily viral hepatitis)

- Effects from the preparatory regimen

- Drug toxicity, including the drugs used for GVHD prophylaxis (cyclosporine and/or methotrexate)

Although the concurrent presence of the characteristic rash provides suggestive clinical evidence, biopsy is the most definitive method to diagnose GVHD of the liver. However, this may not be feasible because of the possibility of acute bleeding due to severe thrombocytopenia soon after HSCT. A transjugular hepatic biopsy may be preferred if an adequate amount of tissue can be obtained. The primary histologic finding is extensive bile duct damage (eg. atypical bile duct and degeneration, epithelial cell dropout, lymphocytic infiltration of small bile ducts), leading to occasionally severe cholestasis.

\section{Acute GVHD diagnosis}

The diagnosis of acute graft-versus-host disease should be considered in any patient who has undergone allogeneic hematopoietic cell transplantation. Acute GVHD can occur at any time point in the post-HSCT setting, but most commonly occurs within the first few months after transplantation or following a reduction of immunosuppression. The diagnosis of acute GVHD can be readily made on clinical grounds alone in the patient who presents with a classic rash, abdominal cramps with diarrhea, and a rising serum bilirubin concentration within the first 100 days following transplantation. In many cases, however, the diagnosis is less straightforward and competing causes for isolated abnormalities must be considered and excluded. Rash alone may be caused by antibiotics or a myriad of other drugs with which these patients are often treated, diarrhea may be infectious in nature, and hyperbilirubinemia may be related to biliary sludge or a side effect of multiple drugs. Histologic confirmation may be helpful to corroborate a clinical impression of possible acute GVHD. The skin and gastrointestinal tract are relatively easy to biopsy. As previously mentioned, percutaneous liver biopsy poses a significant risk of major bleeding since most patients are thrombocytopenic at the time of presentation with GVHD. Transjugular liver biopsy is a safer alternative if it can be adequately performed.

\section{Acute GVHD differential diagnosis}

In most cases, acute graft-versus-host disease (GVHD) is a diagnosis of exclusion and other possible causes of clinical symptoms must be considered. The differential diagnosis depends upon the presenting signs and symptoms of acute GVHD. Most alternative diagnoses can be excluded with biopsy of the involved tissue.

- Skin involvement - The differential diagnosis of patients presenting with signs and symptoms of GVHD of the skin includes other causes of rash, including drug eruptions, viral exanthemas, engraftment syndrome, and radiation dermatitis.

- Gastrointestinal tract - The differential diagnosis of GVHD of the gastrointestinal tract includes other causes of nausea, vomiting, diarrhea, and weight loss. These include infectious causes (Clostridium difficile infection, CMV reactivation), drug effects, chemoradiation toxicity, inflammatory diarrhea, short bowel syndrome, peptic ulcer disease, neoplasms, and systemic disease (eg. diabetes mellitus).

- Liver involvement - The differential diagnosis of hepatic GVHD includes other causes of abnormal liver function tests (increased conjugated bilirubin and alkaline phosphatase). Common confounding disorders also include hepatic sinusoidal obstructive syndrome, hepatic infections (primarily viral hepatitis), effects of the preparatory regimen, and drug toxicity. At times a specific diagnosis is difficult without biopsy.

A continuum of clinical findings may be observed in patients with acute and chronic GVHD, as both disorders commonly affect similar organs, principally the skin, liver, and gastrointestinal tract. However, the target organs affected by, and the clinical and histologic features associated with, chronic GVHD may differ from 
those observed with acute disease. As an example, autoimmune phenomena, such as autoantibody formation, are more common with chronic GVHD. Importantly, diagnostic or distinctive signs of chronic GVHD must be absent in order to make the diagnosis of acute GVHD. Patients with signs or symptoms seen in both entities are considered to have the overlap subtype of chronic GVHD.

\section{Acute GVHD classification}

Acute GVHD is staged and graded based on the degree of organ (skin, liver and gastrointestinal tract) involvement and clinical status of the patient. The degree of skin involvement is graded depending upon the range, degree and severity of the lesions, liver involvement upon the serum total bilirubin $(\mu \mathrm{mol} / \mathrm{l})$ level, and gastrointestinal involvement upon the severity of diarrhea $(\mathrm{ml} /$ day). The clinical features and staging and grading of aGVHD are described in Tables 1, 2 and 3, respectively $(12 ; 13)$. The overall severity of aGVHD has major impact on HSCT outcomes, with transplant-related mortality ranging from 28 for stage 0 to $92 \%$ for stage IV disease (14).

\section{Treatment of aGVHD}

Steroids and calcineurin inhibitor $(\mathrm{CI})$ remain the gold standard for initial treatment of aGVHD (15). Mild skin aGVHD (grade I) can be treated with topical steroids alone. For more severe disease or any visceral involvement (grade II-IV) systemic steroids and CI are the mainstay of treatment. Complete or partial responses are achieved in $44 \%$ patients with improvement in skin, liver and gut disease at 43,35 and $53 \%$, respectively. The response to initial treatment correlates directly with post-transplant survival (16).

The treatment for grade II-IV aGVHD is started with methylprednisolone intravenously (especially in gut GVHD) at $2 \mathrm{mg} / \mathrm{kg}$ /

Tab. 1. Symptoms of acute GVHD.

\begin{tabular}{ll}
\hline Skin & Maculopapular skin rash \\
$\begin{array}{l}\text { Upper } \\
\text { gastrointestinal }\end{array}$ & $\begin{array}{l}\text { Nausea, anorexia, or both, and positive histological } \\
\text { findings }\end{array}$ \\
\hline $\begin{array}{l}\text { Lower } \\
\text { gastrointestinal } \\
\text { tract }\end{array}$ & $\begin{array}{l}\text { Watery diarrhea }(\geq 500 \mathrm{ml}) \\
\text { Severe abdominal pain } \\
\text { Bloody diarrhea or ileus (after exclusion of infectious } \\
\text { causes) }\end{array}$ \\
\hline Liver & Cholestatic hyperbilirubinemia \\
\hline
\end{tabular}

Tab. 3. Overall clinical grading of acute GVHD.

\begin{tabular}{lccc}
\hline Grade & Skin & Liver & Gastrointestinal tract \\
\hline I & Stage 1 & None & None \\
II & Stage 2 & Stage 1-2 or & Stage 1-2 (or both) \\
III & Stage 3 or & Stage 3 or & Stage 3 \\
IV & Stage 4 or & Stage 4 or & Stage 4 \\
\hline
\end{tabular}

day with CI, and continued at that dose 1 to 2 weeks depending on response. If the patient responds well, steroids dose is tapered down to $1.5 \mathrm{mg} / \mathrm{kg}$ / day for 1 week, $1 \mathrm{mg} / \mathrm{kg} /$ day for 1 week, then tapering is continued at the rate of $10 \mathrm{mg} / \mathrm{week}$, and slow down tapering at doses lower than $30 \mathrm{mg} /$ day. Initial steroid dose $1 \mathrm{mg} /$ $\mathrm{kg} /$ day is recommended for aGVHD of the upper GI tract which presents with symptoms of anorexia, nausea/vomiting and dyspepsia. Also in skin GVHD steroid treatment is being started often at a lower dose. Optimal tapering of steroids is over a period of 86-147 days after initial response to treatment, as treatment with steroids especially at higher doses can lead to significant side effects including immunosuppression, hyperglycemia and osteopenia. Many agents (etanercept, mycophenolate, denileukin or pentostatin) in addition to steroids and CI have been evaluated for initial treatment of aGVHD, but they have failed to show significant benefit. Other agents such as basiliximab, daclizumab, antithymocyte globulin (ATG), etanercept and infliximab have also been tested without convincing results (17). Therefore, the addition of agents to high-dose steroids in first-line treatment is only recommended in the setting of clinical trials.

\section{Treatment of steroid refractory aGVHD}

If initial response to steroids is missing and aGVHD worsens in any organ during the first 3 days of steroid treatment or if there is no response during the first 5-14 days, a second agent is added and steroids are tapered $10 \%$ or $10 \mathrm{mg}$ every week from $2 \mathrm{mg} / \mathrm{kg} /$ day dose. The rate of the further tapering depends on the response. The 3 day criterion is especially used for lower GI aGVHD and secondary agents are introduced by fifth day. The decision to add secondline treatment should be made sooner for patients with more severe aGVHD and in patients who cannot tolerate steroid treatment. But none of the existing secondary agents provided convincing evidence for long-term benefits, and the outcome of steroid-refractory aGVHD remains very poor with mortality as high as $80 \%$ (18).

The benefit of antithymocyte globulin (ATG) in steroid-refractory skin aGVHD is when used early. In a prospective randomized

\section{Tab. 2. Organ staging of acute GVHD.}

\begin{tabular}{llcc}
\hline Stage & Skin & $\begin{array}{c}\text { Liver } \\
\text { (bilirubin; } \mu \mathrm{mol} / \mathrm{l})\end{array}$ & Gastrointestinal (GI) (stool output in ml/ day) \\
\hline 0 & No GVHD & $<34$ & $<500 \mathrm{ml}(\mathrm{child}<10 \mathrm{ml} / \mathrm{kg} / \mathrm{day})$ or persistent nausea \\
\hline 1 & Maculopapular rash $<25 \% \mathrm{BSA}$ & $34-50$ & $\begin{array}{l}500-999 \mathrm{ml}(\mathrm{child} 10-19.9 \mathrm{ml} / \mathrm{kg} / \mathrm{day}) \text { or persistent nausea, } \\
\text { vomiting or anorexia with positive upper GI biopsy }\end{array}$ \\
\hline 2 & Maculopapular rash $25-50 \%$ BSA & $51-102$ & $1000-1500 \mathrm{ml}(\mathrm{child} 20-30 \mathrm{ml} / \mathrm{kg} / \mathrm{day})$ \\
\hline 3 & $\begin{array}{l}\text { Maculopapular rash }>50 \% \text { BSA or generalised } \\
\text { erythroderma }\end{array}$ & $103-255$ & $1500-2000 \mathrm{ml}(\mathrm{child}>30 \mathrm{ml} / \mathrm{kg} / \mathrm{day})$ \\
\hline 4 & $\begin{array}{l}\text { Diffuse erythema with bullous formation or } \\
\text { desquamation }\end{array}$ & $>255$ & $>2000 \mathrm{ml}$ or severe abdominal pain, with or without ileus \\
\hline
\end{tabular}

BSA $=$ body surface area 
trial patients with steroid-refractory aGVHD were treated with $5 \mathrm{mg} / \mathrm{kg}$ /day methylprednisolone alone or in combination with rabbit ATG. There was no difference between the two arms in terms of response rates, survival or TRM (19). Alemtuzumab (Campath) is a very potent antibody with $50 \%$ responses even in grade III and IV aGVHD, but CMV reactivation and life-threatening infections occur. Thus caution should be taken not to use too high dose and it should be introduced earlier than later in the course.

Extracorporeal photopheresis (ECP) weekly until maximal disease response achieved CR rates of 82, 61 and $61 \%$ for aGVHD of skin, liver and GI tract, respectively. Transplant-related mortality was only $14 \%$ in patients treated with ECP (20). ECP shows benefit in the treatment of aGVHD, and is safe, without any increase in the rate of infections, secondary malignancies or mortality (21). Mycophenolate mofetil (MMF) treatment of steroidrefractory aGVHD was associated with responses, but this did not translate into long-term overall survival (22). Treatment of steroid-refractory grade III/IV aGVHD with sirolimus was associated with responses in $57 \%$ patients (CR $24 \%$ ), but treatment was discontinued in 10 patients due to no response or toxicity (23). Pentostatin treatment achieved overall response rates greater than $50 \%$ (24). Donor mesenchymal stem cells (MSCs) are helpful in the treatment of steroid-refractory aGVHD and their use in 55 patients was associated with CR in 30 patients and improvement in 9 additional patients (25).

Anti-interleukin 2 receptor antibody daclizumab was given as single second-line agent or added to cyclosporine and mycophenolate, and achieved significant amount of responses (26). Infliximab, a monoclonal antibody that binds to TNF $\alpha$, has been shown to be associated with significant response although the proportion of patients with grade III-IV aGVHD was low and treatment was complicated by aspergillus infections (27). Etanercept, a soluble dimeric TNF $\alpha$ receptor 2 that competes for TNF $\alpha$ binding with cellular receptors, was shown to induce responses in patients with GVHD of the gastrointestinal tract (28). A study of pediatric patients of steroid-refractory aGVHD who were treated with a combination of daclizumab and infliximab showed response in 19 out of 22 patients (29). The published data suggests that treatment with TNF $\alpha$ inhibitors is associated with improved responses in steroid-refractory aGVHD, particularly the ones involving gastrointestinal tract.

\section{Steroid-refractory gut aGVHD}

When patient develops gut GVHD all medications should be changed to IV, particularly CI, for the concern of appropriate absorption, and TPN (total parenteral nutrition) is started. Methylprednisolone intravenously $2 \mathrm{mg} / \mathrm{kg}$ /day should be started, usually divided into twice a day doses. Prophylaxis for bacterial, fungal, Pneumocystis jirovecii and viral infections (acyclovir) should be initiated, or continued. If the patient does not respond to steroid for 3 days, he should start infliximab 5-10 mg/kg weekly $\times 4$ doses. At the same time ECP should be initiated and budesonide should be given orally $3-6 \mathrm{mg}$ daily to 3 times daily. When the patient is responding to the treatment, steroids are tapered first. Usually for these cases steroids are maintained $2 \mathrm{mg} / \mathrm{kg}$ /day for 2 weeks,
Tab. 4. Symptoms of chronic GVHD.

\begin{tabular}{ll}
\hline Skin & $\begin{array}{l}\text { lichen planus-like eruptions, or sclerotic features, } \\
\text { dyspigmentation, new-onset alopecia, poikiloderma }\end{array}$ \\
\hline Nails & dystrophy or loss \\
\hline Eyes & sicca syndrome, dry eyes, cicatrical conjunctivitis \\
\hline Mouth & $\begin{array}{l}\text { ulcers, lichen-type features, xerostomia, restric- } \\
\text { tions of mouth opening from sclerosis }\end{array}$ \\
\hline Muscles, fascia, joints & myositis, fasciitis, or joint stiffness from contractures \\
\hline Gastrointestinal tract & weight loss, anorexia, esophageal web or strictures \\
\hline Liver & jaundice, elevated liver function tests \\
\hline Lungs & $\begin{array}{l}\text { bronchiolitis obliterans, restrictive or obstructive de- } \\
\text { fects on pulmonary function tests, pleural effusions }\end{array}$ \\
\hline Heart & pericarditis \\
\hline Female genitalia & ulcerations, vaginal sclerosis \\
\hline Marrow & thrombocytopenia, anemia,neutropenia, eosinophilia, \\
\end{tabular}

then start tapering $10 \%$ weekly. If diarrhea volume is more than $500 \mathrm{ml} / 24 \mathrm{~h}$ and watery the taper is hold off. When stool volume is less than $500 \mathrm{ml} /$ day and contains some solid particles and is getting "pudding-like", PO intake is initiated, first with clear liquid, then full liquid, then step up the diet very carefully, adding one food item a day. Fat, protein, and dairy products may predispose to diarrhea, so these are food items added last. Treatment for steroid-refractory gut GVHD is a long, painful process, and has high mortality, but it may be possible to save some of these patients by treating them very carefully.

\section{Chronic GVHD classification}

Chronic GVHD is classified as mild, moderate or severe according to the NIH consensus criteria (30). The organs commonly affected by cGVHD include skin, eyes, mouth, liver, gastrointestinal tract, lungs and genitalia. The response to treatment in cGVHD is unpredictable. Mixed responses are seen in different organs in the same patient. The risk factors for the development of cGVHD are similar to aGVHD. The impact of cGVHD on survival must be considered in balance with the fact that cGVHD is associated with lower risk of relapse in leukemia (GVL effect). The correlation between GVHD severity and relapse is unclear (31). The main clinical features are mentioned in Table 4 (32).

\section{Treatment of chronic GVHD}

Mild form of cGVHD often respond to topical treatment with corticosteroids, while systemic therapy is usually needed for treatment of moderate-to-severe cGVHD. Prednisolone $1 \mathrm{mg} / \mathrm{kg} / \mathrm{day}$ alone is usually the first-line treatment. The duration of treatment depends upon response and is often prolonged with median duration 2-3 years (33). Addition of cyclosporine (CSA) (a CI) to steroids lowers the rate of avascular hip necrosis, but not TRM, progression to secondary therapy or duration of immunosuppression. Other agents including MMF have failed to improve results of primary treatment of cGVHD when added to steroids, and are associated with increased mortality (34). Bortezomib in combination with prednisone have been associated with overall response rate of $80 \%$ with very little toxicity (35). 
Progression of cGVHD despite $1 \mathrm{mg} / \mathrm{kg} /$ day of corticosteroids for 2 weeks or lack of improvement in symptoms after 4-8 weeks of continuous therapy or inability to taper corticosteroids are considered as markers of refractory disease (36). There are no standard treatments for steroid-refractory cGVHD. In addition, continuation more than 20-30 mg/day of prednisolone for more than several months is associated with significant toxicities, thus many agents have been tested for steroid-sparing effect. Rituximab has shown response rate of $60 \%$ to $70 \%$ in steroid-refractory cGVHD, but responses were mainly partial and were limited to skin and musculoskeletal disease (37). A recent small prospective study evaluated combination of rituximab with alemtuzumab in 15 patients with steroid-refractory cGVHD (38). The overall response rate was $100 \%$ with 5 patients achieving complete response.

Extracorporeal photopheresis (ECP) and PUVA have shown benefit in the treatment of steroid-refractory or steroid-dependent cGVHD (39). Approximately $50 \%$ patients have reduction in symptoms (40). PUVA is using direct irradiation to the skin and it may be very effective in selected cases with skin cGVHD. The treatment with TKIs (imatinib) appears to be effective particularly in refractory sclerotic cGVHD. Mycophenolate mofetil has shown response rate of $45 \%$ in salvage therapy for cGVHD, but randomized prospective trial of MMF vs placebo in addition to other treatment for cGVHD was terminated early due to no difference in response rate in control and study arms (41). Common side effects of MMF include cytopenias, infections and gastrointestinal toxicity which can mimic aGVHD. Since that study MMF has been less commonly used in the treatment of cGVHD. Sirolimus has been used in combination with tacrolimus and corticosteroids for the treatment of steroid-refractory cGVHD, and overall response rate was $63 \%$ (42). Another retrospective study of patients with severe sclerodermatous cGVHD treated with sirolimus showed a response rate of $76 \%$ (43). Toxicities included thrombotic microangiopathy and renal dysfunction. It is recommended to monitor patients for renal function, hyperlipidemia, myelosuppression particularly thrombocytopenia and thrombotic microangiopathy while on sirolimus. Pentostatin given every other week for a median of 12 doses reported an overall response rate of $55 \%$, despite that most patients were heavily pre-treated (44). Low-dose IL-2 administered daily for 8 weeks induces Treg expansion and achieves partial responses in $50 \%$ of patients, probability and magnitude of response was proportional to the duration of treatment (45). Patients also had improvement in advanced fibrotic and sclerotic manifestations of cGVHD which were previously thought to be irreversible. Bortezomib in a retrospective study of 37 patients with multiple myeloma treated with reduced intensity allogeneic HSCT in 11 patients showed responses with 3 responses in patients with severe cGVHD. Eight patients with limited disease did not require any additional immunosuppressive therapy (46). Thalidomide therapy has shown complete or partial response in patients with refractory cGVHD. Treatment was associated with frequent discontinuation due to toxicity such as neutropenia and neurologic side effects (47). Low dose methotrexate achieves a marked benefit in cutaneous disease in combination with other immunosuppressants (48).

\section{Special considerations in cGVHD treatment}

Severe sclerotic skin cGVHD initial treatment includes a combination of steroid and cyclosporine that may be replaced with sirolimus. Rituximab and ECP should be introduced relatively early. Imatinib should be started at as low as $100 \mathrm{mg}$ every other day, and the dose should be increased until the patient can tolerate it because higher dose may be more effective. Physiotherapy to keep the activity up is a very important part of the treatment. Patients usually have impaired sweating and should be careful to stay in a well air-conditioned room and keep taking a lot of water in summer to avoid heat shock. Blisters and skin infections are treated with oral (doxycycline) and local antibiotics (mupirocin). Patients are at high risk to develop skin cancers, so if they develop suspicious lesions, dermatology consultation must be pursued.

Oral cGVHD is treated with dexamethasone rinse $(0.5 \mathrm{mg} / 5 \mathrm{ml})$ 2-4-times a day (instruct the patient to spit out after rinse, as it may be too much systemic steroid if they swallow it) followed by nystatin swish. Occasionally clobetasol gel application on the erosive lesions is beneficial. Also tacrolimus elixir or sirolimus syrup is used instead of pills to provide respective medications, and the patients are instructed to swish in the mouth before they swallow them.

Most of the patients develop dry eyes, thus artificial tears without preservative are necessary to keep eyes moist. Tear duct plugging has been done to keep eyes as moist as possible and often works well. For more symptomatic patients, cyclosporine and/ or steroid eye drop may be used, but cyclosporine eye drop may irritate the eyes. Eye drops made of autologous serum have been tried and were very effective in some cases (49). Scleral contact lenses, a large size contact lens which rests on sclera and creates a tear-filled vault over the cornea, may help in refractory cases.

A very few effective bronchiolitis obliterans treatments are available (50). Steroids may work partially, but not for a long time, thus steroid should be tapered to a dose the patient can tolerate. Pulmonary rehabilitation is important, and providing support for these patients to change their lifestyle is necessary. For severe cases, lung transplantation may be the only option.

Many patients with cGVHD may be working or would like to be back to work, and it is necessary to support these patients to maintain or find jobs. One should be aware of the transformed self-images particularly female patients with skin GVHD and/or with steroid effect. These patients should be provided with appropriate support including mental aspects. And many patients cannot perform as much as they could before GVHD, thus providing help to accept the situation and set up a new goal is important.

\section{Conclusions}

GVHD is potentially lethal complication and continues to limit survival in patients undergoing HSCT. In the last decade a lot has been learned regarding the mechanisms involved in the pathophysiology of GVHD (51). Recent developments have led to remarkable improvements in the assessment and treatment of patients with GVHD. New technologies have become available to evaluate the extent of the disease. Novel pathways are being tar- 
geted and new agents are being developed/tested for the treatment of GVHD. But a combination of cyclosporin with corticosteroids remains the cornerstone of initial GVHD management. Durable responses with steroids are seen in less than half of the patients treated for aGVHD and about 40-50\% of cGVHD depending upon severity of the disease. Due to the lack of randomized controlled trials for the treatment of steroid-refractory disease, there is no clear consensus on what comprises the best second- and thirdline approach in the treatment of acute and chronic GVHD. Once GVHD is steroid refractory, a large group of potentially effective drugs and procedures have been introduced into clinical practice, which enable clinicians to significantly improve the outcome of patients but also pose new challenges for the prevention and management of their specific side effects. New options need to be considered with great attention paid to the type and stage/grade of GVHD, side effects profile, drug interactions and possible obstacles in administration of the treatment agents. With the current rush in new agents and new findings related to GVHD treatment, we will see a significant advancement in this field in next years. Given these various new options and challenges, it is important to identify the minimal requirements for diagnosis and treatment of GVHD, as access to the most sophisticated advances may vary depending on local circumstances.

\section{References}

1. Griffith LM, Pavletic SZ, Lee SJ, Martin PJ, Schultz KR, Vogelsang GB. Chronic graft-versus-host disease-implementation of the national institutes of health consensus criteria for clinical trials. Biol Blood Marrow Transplant 2008; 14 (4): 379-384.

2. Jagasia MH, Greinix HT, Arora M et al. National Institutes of health consensus development project on criteria for clinical trials in chronic graftversus-host disease: I. The 2014 Diagnosis and Staging Working Group Report. Biol Blood Marrow Transplant 2015; 21 (3): 389-401.

3. Dihenescikova VR, Mistrik M, Martinka J et al. Collection of peripheral hematopoietic stem/progenitor cells. Bratisl Lek Listy 2015; 116 (1): $9-13$.

4. Henden AS, Hill GR. Cytokines in Graft-versus-Host Disease. J Immunol 2015; 194 (10): 4604-4612.

5. Shimabukuro-Vornhagen A, Hallek MJ, Storb RF, von BergweltBaildon MS. The role of B cells in the pathogenesis of graft-versus-host disease. Blood 2009; 114 (24): 4919-4927.

6. Bates JS, Engemann AM, Hammond JM. Clinical utility of rituximab in chronic graft-versus-host disease. Ann Pharmacother 2009; 43 (2): 316-321.

7. Miura Y, Thoburn CJ, Bright EC et al. Association of Foxp3 regulatory gene expression with graft-versus-host disease. Blood 2004; 104 (7): 2187-2193.

8. Cosmi L, Liotta F, Angeli R et al. Th2 cells are less susceptible than Th1 cells to the suppressive activity of $\mathrm{CD} 25+$ regulatory thymocytes because of their responsiveness to different cytokines. Blood 2004; 103 (8): 3117-3121.

9. Auffermann-Gretzinger S, Lossos IS, Vayntrub TA et al. Rapid establishment of dendritic cell chimerism in allogeneic hematopoietic cell transplant recipients. Blood 2002; 99 (4): 1442-1448.
10. Marcondes AM, Karoopongse E, Lesnikova M et al. Alpha-1-antitryp$\sin$ (AAT)-modified donor cells suppress GVHD but enhance the GVL effect: a role for mitochondrial bioenergetics. Blood 2014; 124 (18): 2881-2891.

11. Lowsky R, Takahashi T, Liu YP et al. Protective conditioning for acute graft-versus-host disease. N Engl J Med 2005; 353 (13): 1321-1331.

12. Glucksberg H, Storb R, Fefer A et al. Clinical manifestations of graftversus-host disease in human recipients of marrow from HL-A-matched sibling donors. Transplantation 1974; 18 (4): 295-304.

13. Przepiorka D, Weisdorf D, Martin Pet al.: 1994 Consensus Conference on Acute GVHD Grading. Bone Marrow Transplant 1995; 15 (6): 825-828.

14. Gratwohl A, Hermans J, Apperley J et al. Acute graft-versus-host disease: grade and outcome in patients with chronic myelogenous leukemia. working party chronic leukemia of the European Group for Blood and Marrow Transplantation. Blood 1995; 86 (2): 813-818.

15. Lukas J, Bojtarova E, Mistrik M et al. Treatment difficulty with acute GVHD - frequent cause of mortality after allogeneic hematopoietic stem cell transplantation. Bratisl Lek Listy 2014; 115 (2): 80-82.

16. Saliba RM, Couriel DR, Giralt $S$ et al. Prognostic value of response after upfront therapy for acute GVHD. Bone Marrow Transplant 2012; 47 (1): 125-131.

17. Couriel DR, Saliba R, de Lima M et al. A phase III study of infliximab and corticosteroids for the initial treatment of acute graft-versushost disease. Biol Blood Marrow Transplant 2009; 15 (12): 1555-1562.

18. Weisdorf D, Haake R, Blazar B et al. Treatment of moderate/severe acute graft-versus-host disease after allogeneic bone marrow transplantation: an analysis of clinical risk features and outcome. Blood 1990; 75 (4): $1024-1030$

19. Van Lint MT, Milone G, Leotta S et al. Treatment of acute graftversus-host disease with prednisolone: significant survival advantage for day +5 responders and no advantage for nonresponders receiving antithymocyte globulin. Blood 2006; 107 (10): 4177-4181.

20. Greinix HT, Knobler RM, Worel N et al. The effect of intensified extracorporeal photochemotherapy on long-term survival in patients with severe acute graft-versus-host disease. Haematologica 2006; 91 (3): 405-408.

21. Kitko CL, Levine JE. Extracorporeal photopheresis in prevention and treatment of acute GVHD. Transfus Apher Sci, doi: 10.1016/j.transci.2015.02.001.

22. Furlong T, Martin P, Flowers ME et al. Therapy with mycophenolate mofetil for refractory acute and chronic GVHD. Bone Marrow Transplant 2009; 44 (11): 739-748.

23. Benito AI, Furlong T, Martin PJ et al. Sirolimus (rapamycin) for the treatment of steroid-refractory acute graft-versus-host disease. Transplantation 2001; 72 (12): 1924-1929.

24. Hoda D, Pidala J, Salgado-Vila N et al. Sirolimus for treatment of steroid-refractory acute graft-versus-host disease. Bone Marrow Transplant 2010; 45 (8): 1347-1351.

25. Le Blanc K, Frassoni F, Ball $L$ et al. Mesenchymal stem cells for treatment of steroid-resistant, severe, acute graft-versus-host disease: a phase II study. Lancet 2008; 371 (9624): 1579-1586.

26. Bordigoni P, Dimicoli S, Clement $L$ et al. Daclizumab, an efficient treatment for steroid-refractory acute graft-versus-host disease. Br J Haematol 2006; 135 (3): 382-385.

27. Couriel D, Saliba R, Hicks $K$ et al. Tumor necrosis factor-alpha blockade for the treatment of acute GVHD. Blood 2004; 104 (3): 649-654. 


\section{8-396}

28. Busca A, Locatelli F, Marmont F, Ceretto C, Falda M. Recombinant human soluble tumor necrosis factor receptor fusion protein as treatment for steroid refractory graft-versus-host disease following allogeneic hematopoietic stem cell transplantation. Am J Hematol 2007; 82 (1): 45-52.

29. Rao K, Rao A, Karlsson H, Jagani M, Veys P, Amrolia PJ. Improved survival and preserved antiviral responses after combination therapy with daclizumab and infliximab in steroid-refractory graft-versus-host disease. J Pediatr Hematol Oncol 2009; 31 (6): 456-461.

30. Filipovich AH, Weisdorf D, Pavletic S et al. National institutes of health consensus development project on criteria for clinical trials in chronic graft-versus-host disease: Diagnosis and staging working group report. Biol Blood Marrow Transplant 2005; 11 (12): 945-956.

31. Lee SJ, Klein JP, Barrett AJ et al. Severity of chronic graft-versushost disease: association with treatment-related mortality and relapse. Blood 2002; 100 (2): 406-414.

32. Roziakova L, Bojtarova E, Mistrik M et al. Abnormal cardiomarkers in leukemia patients treated with allogeneic hematopoietic stem cell transplantation. Bratisl Lek Listy 2012; 113 (3): 159-162.

33. Flowers ME, Martin PJ. How we treat chronic graft-versus-host disease. Blood 2015; 125 (4): 606-615.

34. Wolff D, Gerbitz A, Ayuk F et al. Consensus conference on clinical practice in chronic graft-versus-host disease (GVHD): first-line and topical treatment of chronic GVHD. Biol Blood Marrow Transplant 2010; 16 (12): $1611-1628$.

35. Herrera AF, Kim HT, Bindra B et al. A phase II study of bortezomib plus prednisone for initial therapy of chronic graft-versus-host disease. Biol Blood Marrow Transplant 2014; 20 (11): 1737-1743.

36. Martin PJ, Weisdorf D, Przepiorka D et al. National institutes of health consensus development project on criteria for clinical trials in chronic graft-versus-host disease: VI. design of clinical trials working group report. Biol Blood Marrow Transplant 2006; 12 (5): 491-505.

37. Cutler C, Miklos D, Kim HT et al. Rituximab for steroid-refractory chronic graft-versus-host disease. Blood 2006; 108 (2): 756-762.

38. Gutierrez-Aguirre CH, Cantu-Rodriguez OG, Borjas-Almaguer OD et al. Effectiveness of subcutaneous low-dose alemtuzumab and rituximab combination therapy for steroid-resistant chronic graft-versus-host disease. Haematologica 2012; 97 (5): 717-722.

39. Flowers ME, Apperley JF, van Besien K et al. A multicenter prospective phase 2 randomized study of extracorporeal photopheresis for treatment of chronic graft-versus-host disease. Blood 2008; 112 (7): 2667-2674.
40. Dignan FL, Greenblatt D, Cox M et al. Efficacy of bimonthly extracorporeal photopheresis in refractory chronic mucocutaneous GVHD. Bone Marrow Transplant 2012; 47 (6): 824-830.

41. Martin PJ, Storer BE, Rowley SD et al. Evaluation of mycophenolate mofetil for initial treatment of chronic graft-versus-host disease. Blood 2009; 113 (21): 5074-5082.

42. Couriel DR, Saliba R, Escalon MP et al. Sirolimus in combination with tacrolimus and corticosteroids for the treatment of resistant chronic graft-versus-host disease. Br J Haematol 2005; 130 (3): 409-417.

43. Jedlickova Z, Burlakova I, Bug G, Baurmann H, Schwerdtfeger $\mathbf{R}$, Schleuning $\mathbf{M}$. Therapy of sclerodermatous chronic graft-versus-host disease with mammalian target of rapamycin inhibitors. Biol Blood Marrow Transplant 2011; 17 (5): 657-663.

44. Jacobsohn DA, Chen AR, Zahurak M et al. Phase II study of pentostatin in patients with corticosteroid-refractory chronic graft-versus-host disease. J Clin Oncol 2007; 25 (27): 4255-4261.

45. Koreth J, Matsuoka K, Kim HT et al. Interleukin-2 and regulatory T cells in graft-versus-host disease. N Engl J Med 2011; 365 (22): 2055-2066.

46. El-Cheikh J, Michallet M, Nagler A et al. High response rate and improved graft-versus-host disease following bortezomib as salvage therapy after reduced intensity conditioning allogeneic stem cell transplantation for multiple myeloma. Haematologica 2008; 93 (3): 455-458.

47. Koc S, Leisenring W, Flowers ME et al. Thalidomide for treatment of patients with chronic graft-versus-host disease. Blood 2000; 96 (12): 3995-3996.

48. Wang Y, Xu LP, Liu DH et al. First-line therapy for chronic graftversus-host disease that includes low-dose methotrexate is associated with a high response rate. Biol Blood Marrow Transplant 2009; 15 (4): 505-511.

49. Rocha EM, Pelegrino FS, de Paiva CS, Vigorito AC, de Souza CA. GVHD dry eyes treated with autologous serum tears. Bone Marrow Transplant 2000; 25 (10): 1101-1103.

50. Arora M, Cutler CS, Jagasia MH et al. Late Acute and Chronic Graft-versus-Host Disease after Allogeneic Hematopoietic Cell Transplantation. Biol Blood Marrow Transplant, article in press, doi: 10.1016/j. bbmt.2015.10.018.

51. Teshima T, Reddy P, Zeiser R. Acute Graft-versus-Host Disease: Novel Biological Insights. Biol Blood Marrow Transplant, article in press, doi: 10.1016/j.bbmt.2015.10.001.

Received November 21, 2015. Accepted December 1, 2015. 